\title{
Transmission and reflection of terahertz plasmons in two-dimensional plasmonic devices
}

\author{
Oleksiy Sydoruk, Kaushal Choonee, and Gregory C. Dyer
}

\begin{abstract}
Plasmons in two-dimensional semiconductor devices will be reflected by discontinuities, notably, junctions between gated and non-gated electron channels. The transmitted and reflected plasmons can form spatially- and frequency-varying signals, and their understanding is important for the design of terahertz detectors, oscillators, and plasmonic crystals. Using mode decomposition, we studied terahertz plasmons incident on a junction between a gated and a non-gated channel. The plasmon reflection and transmission coefficients were found numerically and analytically, and studied between 0.3 and $1 \mathrm{THz}$ for a range of electron densities. At higher frequencies, we could describe the plasmons by a simplified model of channels in homogeneous dielectrics, for which the analytical approximations were accurate. At low frequencies, however, the full geometry and mode spectrum had to be taken into account. The results agreed with simulations by the finite-element method. Mode decomposition thus proved to be a powerful method for plasmonic devices, combining the rigor of complete solutions of Maxwell's equations with the convenience of analytical expressions.
\end{abstract}

Index Terms-plasmon, two-dimensional channel, 2DEG, mode decomposition

\section{INTRODUCTION}

$\mathbf{T}$ WO-DIMENSIONAL semiconductor structures that support plasmons offer several applications for the devicestarved terahertz band. Plasmon nonlinearities can be used in detectors [1]-[4], oscillators [5]-[9], mixers [1], [10], [11], modulators [12], and plasmonic crystals [13], [14]. Also, plasmons can be coupled to electromagnetic waves by grating couplers and antennas, and their frequencies can be tuned in a wide range by a dc voltage. On the other hand, graphene combines the above properties with the promise of roomtemperature operation [15], [16].

A typical plasmonic structure may contain several sections of gated and non-gated electron channels, see Fig. 1(a). The channels are embedded in a dielectric, which either interfaces with air or is closed by a gate. The gate can control the electron density in a channel, and a periodic array of gates can constitute a grating coupler. Because of the different geometries and electron densities, plasmons in the gated and non-gated channels may have different wavenumbers and field distributions. As a result, plasmons will partially reflect and partially transmit when incident on the junction between two sections. The reflected and transmitted plasmons will

O. Sydoruk is with the Department of Electrical and Electronic Engineering, Imperial College London, SW7 2AZ London, UK email: osydoruk@imperial.ac.uk

K. Choonee is with the National Physical Laboratory, Hampton Road, Teddington TW11 0LW UK email: kaushal.choonee@npl.co.uk

G. C. Dyer is with the Sandia National Laboratories, P.O. Box 5800, Albuquerque, New Mexico 87185, USA email: gcdyer@sandia.gov

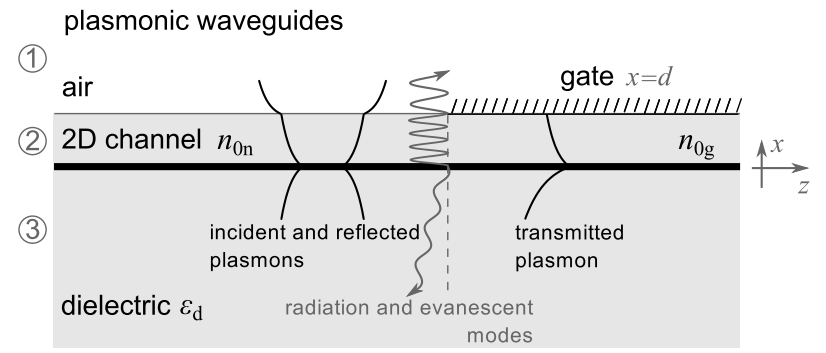

(a)

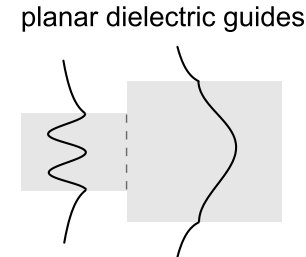

(b) parallel-plate guides

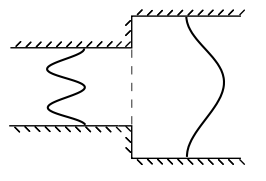

(c)
Fig. 1. Plasmons propagating in gated and non-gated electron channels have different field distributions (a). A plasmon incident at the junction will give rise to a reflected and a transmitted plasmon. In addition, radiation and evanescent modes will be excited. Plasmon reflection and transmission is analogous to those of waves in dielectric (b) and metallic (c) waveguide, and can be described using the same methods.

form frequency- and spatially-varying amplitude distributions, providing a basis for resonators, oscillators, and plasmonic crystals [9], [13], [17].

Design of plasmonic devices requires, therefore, understanding of how plasmons behave at the junctions. From the electromagnetics point of view, the problem is analogous to that of discontinuities in open dielectric [18]-[20] and closed metallic [21] waveguides, see Figs. 1(b) and (c). Naturally, the methods developed during decades of research in waveguides could also be applied for plasmonic structures. And indeed, mode decomposition has been used to study plasmons in metals at optical frequencies [22], [23], and more recently, for two-dimensional $\mathrm{THz}$ plasmons in the presence of a dc current [9], [24]-[27].

Two-dimensional structures were also studied using the Fourier analysis [28], a transmission-line model [13], [14], [29], and finite-difference-time-domain simulations [30], [31]. Although mode-decomposition techniques have been overlooked for passive structures, they can offer several advantages. In contrast to the transmission-line model of Refs. [13], [14], [29], mode-decomposition relies on rigorous solutions 
of Maxwell's equations, considers the plasmon fields in the dielectric as well as in the channel, and takes non-plasmonic modes into account. On the other hand, compared to fullwave numerical simulations, mode-decomposition techniques offer the advantage of fast calculations and even analytical approximations.

Using mode decomposition, this paper analyzes junctions between a gated and non-gated electron channels. Section II introduces the geometry and the model, and discusses the mode spectra. Section III formulates the reflection problem, and Section IV presents its solution by several approaches. Section V draws conclusions.

\section{GEOMETRY AND MODE SPECTRA}

This section presents the plasmonic waveguide configuration and discusses its eigenmodes. A two-dimensional electron channel, placed at $x=0$, is embedded in a dielectric with the relative permittivity $\varepsilon_{\mathrm{d}}$, see Fig. 1(a). The dielectric is unbounded below the channel. For the non-gated structure, the dielectric interfaces with air at $x=d$. For the gated structure, the dielectric is terminated at $x=d$ by an electrically conducting gate. Air is also above the gate. The non-gated and gated channels can have different electron densities, $n_{0 \mathrm{n}}$ and $n_{0 \mathrm{~g}}$, respectively.

For a TM electromagnetic wave, with the amplitudes of the field components $E_{x}, E_{z}$ and $H_{y}$, the electron dynamics in the channels can be described by Newton's equation of motion as

$$
\mathrm{j} \omega v=\frac{e}{m} E_{z}
$$

where $v$ is the amplitude of the ac electron velocity, $\omega$ is the angular frequency, $e$ is the electron charge, and $m$ is the effective electron mass. Denoting the longitudinal wavenumber by $k_{z}$ and the transverse wavenumbers in air and dielectric by $k_{x \mathrm{a}}$ and $k_{x \mathrm{~d}}$, respectively, the dispersion relations are

$$
\begin{aligned}
& k_{z}^{2}+k_{x \mathrm{a}}^{2}=k_{0}^{2} \\
& k_{z}^{2}+k_{x \mathrm{~d}}=k_{0}^{2} \varepsilon_{\mathrm{d}}
\end{aligned}
$$

Here $k_{0}=\omega / c$ is the free-space wavenumber with $c$ denoting the vacuum light velocity. The fields obey the standard boundary conditions. At $x=0$, these are

$$
\begin{aligned}
& \left.E_{z}^{(2)}\right|_{x=0}=\left.E_{z}^{(3)}\right|_{x=0} \\
& \left.E_{x}^{(2)}\right|_{x=0}-\left.E_{x}^{(3)}\right|_{x=0}=\frac{e n}{\varepsilon_{0} \varepsilon_{\mathrm{d}}} \\
& \left.H_{y}^{(2)}\right|_{x=0}-\left.H_{y}^{(3)}\right|_{x=0}=J
\end{aligned}
$$

where $n$ and $J$ are the amplitudes of the ac charge and current density, respectively, and $\varepsilon_{0}$ is the free-space permittivity. The superscripts (1), (2), and (3) correspond to the space in the air, in the dielectric above the channel, and in the dielectric below it, respectively, see Fig. 1(a). The ac current density is $J=e n_{0} v$, where $n_{0}$ is the dc electron density. At $x=d$ and for the non-gated channel, the boundary conditions are

$$
\begin{aligned}
& \left.E_{z}^{(1)}\right|_{x=d}=\left.E_{z}^{(2)}\right|_{x=d} \\
& \left.H_{y}^{(1)}\right|_{x=d}=\left.H_{y}^{(2)}\right|_{x=d}
\end{aligned}
$$

The corresponding condition for the gated channel is $\left.E_{z}^{(1,2)}\right|_{x=d}=0$.
As is well-known, both gated and non-gated channels can support plasmons, whose amplitudes decay away from the channels. Their field distributions are different in the gated and non-gated waveguides, and therefore, a plasmon incident on the junction will give rise to a reflected and a transmitted plasmon. However, $E_{x}$ and $H_{y}$ fields should match at the junction, which cannot be achieved solely by the plasmons. As a result, other, non-plasmonic, eigenmodes will be excited. These modes do not obey the plasmonic dispersion relation and are not bound to the channel.

The situation is analogous to discontinuities in traditional dielectric and metallic waveguides, see Fig. 1(b) and (c). In the former, an incident wave will excite an infinite number of reflected and transmitted waves that can be propagating or evanescent. In the latter, an incident surface wave could excite other surface waves as well as radiation and evanescent modes.

To solve the reflection problem one has, therefore, to know the complete mode spectrum of the waveguide. Plasmonic waveguides, like dielectric ones, support modes with a discrete and a continuous spectrum, as discussed next.

\section{A. Discrete spectrum: Plasmons}

Both gated and non-gated channels can support a single plasmon. The plasmon wavenumbers are governed by dispersion relations, derived assuming an exponential field decay away from a channel, using the equation of motion Eq. (1), and applying the boundary conditions Eq. (3) and (4).

1) Non-gated waveguides: The plasmon dispersion relation for non-gated waveguides can be written as (see e. g. [32])

$$
\left(1-\Gamma_{\mathrm{n}}\right)(1+\zeta) \mathrm{e}^{-\mathrm{j} k_{x \mathrm{~d}} d}-\Gamma_{\mathrm{n}}(1-\zeta) \mathrm{e}^{\mathrm{j} k_{x \mathrm{~d}} d}=0
$$

where $\Gamma_{\mathrm{n}}=-\mathrm{jen} n_{0 \mathrm{n}} k_{x \mathrm{~d}} /\left(2 m \varepsilon_{0} \varepsilon_{\mathrm{d}} \omega^{2}\right)$ and $\zeta=k_{x \mathrm{~d}} /\left(k_{x \mathrm{a}} \varepsilon_{\mathrm{d}}\right)$. Solved with Eq. (2), Eq. (5) gives at a chosen frequency a single solution with real $k_{z}$ and imaginary $k_{x}$. The solid line in Fig. 2 shows the dispersion curve for a GaAs channel ( $m=$ $0.067 m_{0}, \varepsilon_{\mathrm{d}}=12.8, m_{0}$ is the free electron mass) with $n_{0 \mathrm{n}}=$ $4.14 \times 10^{11} \mathrm{~cm}^{-2}$ and $d=400 \mathrm{~nm}$ (corresponding to recent experiments by Dyer et al. [13], [14]).

If the dielectric above the channel extended to infinity, then $\zeta=1$, and the dispersion relation would simplify to the standard expression $\Gamma_{\mathrm{n}}=1$. The role of the dielectricair interface at $x=d$ depends on the dielectric thickness $d$, the dc electron density, and the frequency. The dashed line in Fig. 2 shows the dispersion curve assuming a homogeneous dielectric around the channel. It differs from the complete solution of Eq. (5), especially at lower frequencies; at $0.6 \mathrm{THz}$, the difference between the plasmon wavenumbers is around $30 \%$.

The amplitudes of, for example, the $H_{y}$ component can be written as

$$
\begin{aligned}
& H_{y \mathrm{n}}^{(1)}=-A_{\mathrm{n}} \Gamma_{\mathrm{n}} \mathrm{e}^{\mathrm{j} k_{x \mathrm{~d}} d} \frac{2 \zeta}{1+\zeta} \mathrm{e}^{\mathrm{j} k_{x \mathrm{a}}(x-d)} \\
& H_{y \mathrm{n}}^{(2)}=A_{\mathrm{n}}\left(1-\Gamma_{\mathrm{n}}\right) \mathrm{e}^{-\mathrm{j} k_{x \mathrm{~d}} x}-\Gamma_{\mathrm{n}} \mathrm{e}^{\mathrm{j} k_{x \mathrm{~d}} x} \\
& H_{y \mathrm{n}}^{(3)}=A_{\mathrm{n}} \mathrm{e}^{-\mathrm{j} k_{x \mathrm{~d}} x}
\end{aligned}
$$

where $A_{\mathrm{n}}$ is a constant. The corresponding $E_{x}$ and $E_{z}$ components can be readily found from Maxwell's equations. 


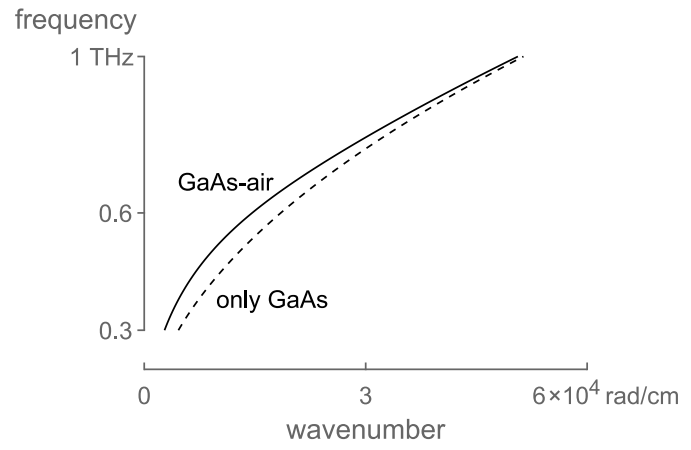

Fig. 2. The plasmon dispersion in a non-gated channel depends on the GaAsair interface (solid line). It is different for homogeneous GaAs (dashed line).

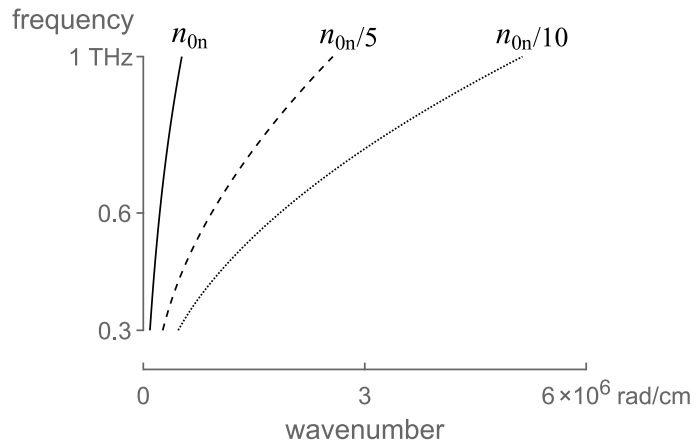

Fig. 3. The dispersion of the gated plasmons depends on the dc electron density, controlled by the gate voltage.

2) Gated waveguides: The dispersion relation for the plasmons in the gated channels is

$$
\Gamma_{\mathrm{g}} \mathrm{e}^{\mathrm{j} k_{x \mathrm{~d}} d}+\left(1-\Gamma_{\mathrm{g}}\right) \mathrm{e}^{-\mathrm{j} k_{x \mathrm{~d}} d}=0
$$

where $\Gamma_{\mathrm{g}}=-\mathrm{jen} n_{0 \mathrm{~g}} k_{x \mathrm{~d}} /\left(2 m \varepsilon_{0} \varepsilon_{\mathrm{d}} \omega^{2}\right)$. A dc voltage applied between the gate and the channel can control the dc electron density $n_{0 \mathrm{~g}}$ and, hence, the plasmon dispersion. Figure 3 shows three dispersion curves for $n_{0 \mathrm{~g}}=n_{0 \mathrm{n}}$ (solid line), $n_{0 \mathrm{~g}}=n_{0 \mathrm{n}} / 5$ (dashed line), and $n_{0 \mathrm{~g}}=n_{0 \mathrm{n}} / 10$ (dotted line), where the value of $n_{0 \mathrm{n}}$ is the same as in Fig. 2, $4.14 \times 10^{12} \mathrm{~cm}^{-2}$.

The amplitudes of the $H_{y}$ field are in the form

$$
\begin{aligned}
& H_{y \mathrm{~g}}^{(2)}=-\frac{\mathrm{j} A_{\mathrm{g}} \cos \left[k_{x \mathrm{~d}}(x-d)\right]}{\sin \left(k_{x \mathrm{~d}} d\right)} \\
& H_{y \mathrm{~g}}^{(3)}=A_{\mathrm{g}} \mathrm{e}^{-\mathrm{j} k_{x \mathrm{~d}} x}
\end{aligned}
$$

where $A_{\mathrm{g}}$ is a constant.

\section{B. Continuous spectrum: Radiation and evanescent modes}

Apart from plasmons, both configurations also support bulk modes, whose amplitudes remain finite far from the channels. The modes can have arbitrary real values of the transverse wavenumber $k_{x}$, constituting a continuous spectrum. The corresponding longitudinal wavenumbers $k_{z}$, found from the dispersion relations, Eq. (2), are either real (radiation modes) or imaginary (evanescent modes).

For the asymmetric geometries of Fig. 1(a), the spectrum can be conveniently separated into bulk 'top' and 'bottom' modes [22]. The top modes of the non-gated waveguide are found assuming a plane wave incident from the top, a reflected wave in the opposite direction, and a transmitted wave, see Fig. 4(a). The bottom modes are found analogously, assuming incidence from the bottom, see Fig. 4(b). The top and bottom modes of the gated waveguide do not have a transmitted wave, see Figs. 4(c) and (d).

1) Non-gated waveguides: The $H_{y}$ components for the top modes of a non-gated waveguide can be written in the form

$$
\begin{aligned}
& h_{y \mathrm{nt}}^{(1)}=\frac{a_{\mathrm{nt}}}{2}\left(A \mathrm{e}^{\mathrm{j} k_{x \mathrm{a}}(x-d)}+B \mathrm{e}^{-\mathrm{j} k_{x \mathrm{a}}(x-d)}\right) \\
& h_{y \mathrm{nt}}^{(2)}=a_{\mathrm{nt}}\left(C \mathrm{e}^{\mathrm{j} k_{x \mathrm{~d}} x}+D \mathrm{e}^{-\mathrm{j} k_{x \mathrm{~d}} x}\right) \\
& h_{y \mathrm{nt}}^{(3)}=a_{\mathrm{nt}} \mathrm{e}^{-\mathrm{j} k_{x \mathrm{~d}} x}
\end{aligned}
$$

where $a_{\mathrm{nt}}$ is a constant, and $C=-\Gamma_{\mathrm{n}}, D=1-\Gamma_{\mathrm{n}}$ and

$$
\begin{aligned}
& A=\left(1-\Gamma_{\mathrm{n}}\right)(1-\zeta) \mathrm{e}^{-\mathrm{j} k_{x \mathrm{~d}} d}-\Gamma_{\mathrm{n}}(1+\zeta) \mathrm{e}^{\mathrm{j} k_{x \mathrm{~d}} d} \\
& B=\left(1-\Gamma_{\mathrm{n}}\right)(1+\zeta) \mathrm{e}^{-\mathrm{j} k_{x \mathrm{~d}} d}-\Gamma_{\mathrm{n}}(1-\zeta) \mathrm{e}^{\mathrm{j} k_{x \mathrm{~d}} d}
\end{aligned}
$$

On the other hand, the bottom modes can be presented in the form

$$
\begin{aligned}
& h_{y \mathrm{nb}}^{(1)}=a_{\mathrm{nb}} \mathrm{e}^{\mathrm{j} k_{x \mathrm{a}}(x-d)} \\
& h_{y \mathrm{nb}}^{(2)}=\frac{a_{\mathrm{nb}}}{2 \zeta}\left(A \mathrm{e}^{\mathrm{j} k_{x \mathrm{~d}}(x-d)}-B \mathrm{e}^{-\mathrm{j} k_{x \mathrm{~d}}(x-d)}\right) \\
& h_{y \mathrm{nb}}^{(3)}=\frac{a_{\mathrm{nb}}}{2 \zeta}\left(C \mathrm{e}^{\mathrm{j} k_{x \mathrm{~d}} x}-D \mathrm{e}^{-\mathrm{j} k_{x \mathrm{~d}} x}\right)
\end{aligned}
$$

where $a_{\mathrm{nb}}$ is a constant, and $A=1+\zeta, B=1-\zeta$ and

$$
\begin{aligned}
& C=(1+\zeta)\left(1-\Gamma_{\mathrm{n}}\right) \mathrm{e}^{-\mathrm{j} k_{x \mathrm{~d}} d}-(1-\zeta) \Gamma_{\mathrm{n}} \mathrm{e}^{\mathrm{j} k_{x \mathrm{~d}} x} \\
& D=(1+\zeta) \Gamma_{\mathrm{n}} \mathrm{e}^{-\mathrm{j} k_{x \mathrm{~d}} d}-(1-\zeta)\left(1+\Gamma_{\mathrm{n}}\right) \mathrm{e}^{\mathrm{j} k_{x \mathrm{~d}} x}
\end{aligned}
$$

2) Gated waveguides: The $H_{y}$ components for the top modes of a gated waveguide can be written in the form

$$
h_{y \mathrm{gt}}^{(1)}=a_{\mathrm{gt}} \cos \left[k_{x \mathrm{a}}(x-d)\right]
$$

where $a_{\mathrm{gt}}$ is a constant. On the other hand, the bottom modes can be presented in the form

$$
\begin{aligned}
& h_{y \mathrm{gb}}^{(2)}=2 a_{\mathrm{gb}} \cos \left[k_{x \mathrm{~d}}(x-d)\right] \\
& h_{y \mathrm{gb}}^{(3)}=a_{\mathrm{gb}}\left(A \mathrm{e}^{\mathrm{j} k_{x \mathrm{~d}} x}+B \mathrm{e}^{-\mathrm{j} k_{x \mathrm{~d}} x}\right)
\end{aligned}
$$

where $a_{\mathrm{gb}}$ is a constant and

$$
\begin{aligned}
& A=\Gamma_{\mathrm{g}} \mathrm{e}^{\mathrm{j} k_{x \mathrm{~d}} d}+\left(1-\Gamma_{\mathrm{g}}\right) \mathrm{e}^{-\mathrm{j} k_{x \mathrm{~d}} d} \\
& B=\left(1+\Gamma_{\mathrm{g}}\right) \mathrm{e}^{\mathrm{j} k_{x \mathrm{~d}} d}-\Gamma_{\mathrm{g}} \mathrm{e}^{-\mathrm{j} k_{x \mathrm{~d}} d}
\end{aligned}
$$

\section{Mode orthogonality, overlap, and power}

Almost all mode-decomposition techniques rely on the property of mode orthogonality. In the non-gated waveguide, for example, the plasmon and any bulk mode are orthogonal, 


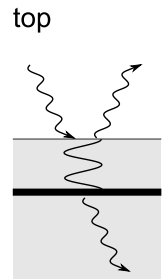

(a)

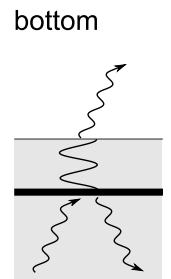

(b)

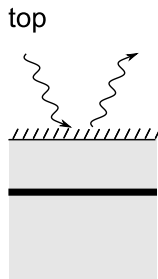

(c) bottom

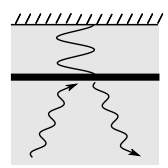

(d)

Fig. 4. The bulk modes of both gated and non-gated waveguides can be separated into top, (a) and (c), and bottom, (b) and (d), modes. The top modes are obtained assuming a plane wave incident from above, and the bottom ones, from below. The top and bottom modes of the non-gated waveguide extend everywhere in space, whereas those of the gated waveguide are separated by the gate.

which can be written introducing the following scalar product as

$$
\begin{aligned}
\left\langle H_{y \mathrm{n}} h_{y \mathrm{nt}, \mathrm{b}}\right\rangle & =\int_{d}^{\infty} H_{y \mathrm{n}}^{(1)} h_{y \mathrm{nt}, \mathrm{b}}^{(1)} d x \\
& +\frac{1}{\varepsilon_{\mathrm{d}}} \int_{0}^{d} H_{y \mathrm{n}}^{(2)} h_{y \mathrm{nt}, \mathrm{b}}^{(2)} d x \\
& +\frac{1}{\varepsilon_{\mathrm{d}}} \int_{-\infty}^{0} H_{y \mathrm{n}}^{(3)} h_{y \mathrm{nt}, \mathrm{b}}^{(3)} d x=0
\end{aligned}
$$

The plasmon and any bulk mode are also orthogonal in the gated waveguide, which can be written, introducing a similar scalar product for the gated channel, as

$$
\begin{aligned}
& \left\langle H_{y \mathrm{~g}} h_{y \mathrm{gb}}\right\rangle= \\
& \frac{1}{\varepsilon_{\mathrm{d}}} \int_{0}^{d} H_{y \mathrm{~g}}^{(2)} h_{y \mathrm{gb}}^{(2)} d x+\frac{1}{\varepsilon_{\mathrm{d}}} \int_{-\infty}^{0} H_{y \mathrm{~g}}^{(3)} h_{y \mathrm{gb}}^{(3)} d x=0
\end{aligned}
$$

On the other hand, the orthogonality conditions for two bulk modes with the transverse wavenumbers $k_{x}$ and $\tilde{k}_{x}$ can be written in the form

$$
\begin{aligned}
& \left\langle h_{y \mathrm{nt}}\left(k_{x}\right) h_{y \mathrm{nt}}\left(\tilde{k}_{x}\right)\right\rangle=S_{\mathrm{ntt}} \delta\left(k_{x}-\tilde{k}_{x}\right) \\
& \left\langle h_{y \mathrm{nb}}\left(k_{x}\right) h_{y \mathrm{nb}}\left(\tilde{k}_{x}\right)\right\rangle=S_{\mathrm{nbb}} \delta\left(k_{x}-\tilde{k}_{x}\right) \\
& \left\langle h_{y \mathrm{nt}}\left(k_{x}\right) h_{y \mathrm{nb}}\left(\tilde{k}_{x}\right)\right\rangle=S_{\mathrm{ntb}} \delta\left(k_{x}-\tilde{k}_{x}\right) \\
& \left\langle h_{y \mathrm{gt}}\left(k_{x}\right) h_{y \mathrm{gt}}\left(\tilde{k}_{x}\right)\right\rangle=S_{\mathrm{gtt}} \delta\left(k_{x}-\tilde{k}_{x}\right) \\
& \left\langle h_{y \mathrm{gb}}\left(k_{x}\right) h_{y \mathrm{gb}}\left(\tilde{k}_{x}\right)\right\rangle=S_{\mathrm{gbb}} \delta\left(k_{x}-\tilde{k}_{x}\right)
\end{aligned}
$$

where $S_{\mathrm{ntt}}, S_{\mathrm{nbb}}, S_{\mathrm{ntb}}, S_{\mathrm{gtt}}, S_{\mathrm{gbb}}$ are constants, and $\delta$ is the Dirac delta function. The scalar products in Eq. (15) are the same as in Eqs. (13) and (14), and $\left\langle h_{y \text { gt }}\left(k_{x}\right) h_{y \text { gt }}\left(\tilde{k}_{x}\right)\right\rangle=$ $\int_{d}^{\infty} h_{y \mathrm{gt}}^{(1)}\left(k_{x}\right) h_{y \mathrm{gt}}^{(1)}\left(\tilde{k}_{x}\right) d x$. All constants except $S_{\text {ntb }}$ can be made equal to unity by a choice of $a_{\mathrm{nt}}, a_{\mathrm{nb}}, a_{\mathrm{gt}}$, and $a_{\mathrm{gb}}$ in Eqs. (9)-(12). The same is true for $\left\langle H_{y \mathrm{n}} H_{y \mathrm{n}}\right\rangle=S_{\mathrm{np}}$ and $\left\langle H_{y \mathrm{~g}} H_{y \mathrm{~g}}\right\rangle=S_{\mathrm{gp}}$.

On the other hand, pairs of modes from different waveguides are not generally orthogonal. The mode overlap can be defined using the same scalar products as in Eqs. (13) and (14). For

example, the overlap between the two plasmons is of the form

$$
\begin{aligned}
J_{\mathrm{pp}}=\left\langle H_{y \mathrm{n}} H_{y \mathrm{~g}}\right\rangle= & \frac{1}{\varepsilon_{\mathrm{d}}} \int_{0}^{d} H_{y \mathrm{n}}^{(2)} H_{y \mathrm{~g}}^{(2)} d x \\
& +\frac{1}{\varepsilon_{\mathrm{d}}} \int_{-\infty}^{0} H_{y \mathrm{n}}^{(3)} H_{y \mathrm{~g}}^{(3)} d x,
\end{aligned}
$$

and the overlap between the top modes is

$$
\begin{aligned}
J_{\mathrm{tt}}\left(k_{x}, \tilde{k}_{x}\right) & =\left\langle h_{y \mathrm{nt}}\left(k_{x}\right) h_{y \mathrm{gt}}\left(\tilde{k}_{x}\right)\right\rangle \\
& =\int_{d}^{\infty} h_{y \mathrm{nt}}^{(1)}\left(k_{x}\right) h_{y \mathrm{gt}}^{(1)}\left(\tilde{k}_{x}\right) d x
\end{aligned}
$$

Similarly, $J_{\mathrm{tp}}\left(k_{x}\right)$ denotes the overlap between a top mode from the non-gated and the plasmon of the gated waveguide, $J_{\mathrm{pt}}\left(k_{x}\right)$ the overlap between the plasmon from the gated waveguide and a top mode from the non-gated one. The remaining overlaps are denoted as $J_{\mathrm{bp}}, J_{\mathrm{pb}}, J_{\mathrm{bb}}, J_{\mathrm{tb}}$, and $J_{\mathrm{bt}}$, where the first letter of the subscript denotes the mode from the non-gated waveguide (plasmon, top, or bottom) and the second letter, the mode from the gated one.

The power carried by a mode can be found using the standard expression $1 / 2 \operatorname{Re} \int_{-\infty}^{\infty} H_{y} E_{x}^{*} \mathrm{~d} x$, where the asterisk denotes complex conjugation. It is non-zero only for the modes with real $k_{z}$ (plasmons and radiation modes), for which it is proportional to the scalar self-products $S$ defined above.

\section{FIELDS AT THE JUNCTION}

The complete mode spectra are now known and can be used to present the fields in both waveguides. Assuming a single incident plasmon in the non-gated waveguide, the total field there will be a superposition of the incident plasmon, the reflected plasmon, and the bulk top and bottom modes. The field in the gated waveguide will be a superposition of the transmitted plasmon and the bulk modes. The $H_{y}$ and $E_{x}$ fields should match at the junction, and the boundary conditions for the magnetic field can be written as

$$
\begin{aligned}
& H_{y \mathrm{n}}^{(1)}+R H_{y \mathrm{n}}^{(1)}+\int_{0}^{\infty} r_{\mathrm{nt}}\left(k_{x}\right) h_{y \mathrm{nt}}^{(1)}\left(k_{x}\right) \mathrm{d} k_{x} \\
& +\int_{0}^{\infty} r_{\mathrm{nb}}\left(k_{x}\right) h_{y \mathrm{nb}}^{(1)}\left(k_{x}\right) \mathrm{d} k_{x} \\
& =\int_{0}^{\infty} t_{\mathrm{gt}}\left(k_{x}\right) h_{y \mathrm{gt}}^{(1)}\left(k_{x}\right) \mathrm{d} k_{x}
\end{aligned}
$$

in the air and

$$
\begin{aligned}
& H_{y \mathrm{n}}^{(2,3)}+R H_{y \mathrm{n}}^{(2,3)}+\int_{0}^{\infty} r_{\mathrm{nt}}\left(k_{x}\right) h_{y \mathrm{nt}}^{(2,3)}\left(k_{x}\right) \mathrm{d} k_{x} \\
& +\int_{0}^{\infty} r_{\mathrm{nb}}\left(k_{x}\right) h_{y \mathrm{nb}}^{(2,3)}\left(k_{x}\right) \mathrm{d} k_{x} \\
& =T H_{y \mathrm{~g}}^{(2,3)}+\int_{0}^{\infty} t_{\mathrm{gb}}\left(k_{x}\right) h_{y \mathrm{gb}}^{(2,3)}\left(k_{x}\right) \mathrm{d} k_{x}
\end{aligned}
$$

in the dielectric. Here, the amplitude of the incident plasmon is unity, $R$ is the plasmon (amplitude) reflection coefficient, 
$T$ is the plasmon transmission coefficient, and $r_{\mathrm{nt}}, r_{\mathrm{nb}}, t_{\mathrm{gt}}$, $t_{\mathrm{gb}}$ are the reflection and transmission coefficients of the bulk modes.

The boundary conditions for the electric field are of the form

$$
\begin{aligned}
& k_{z \mathrm{np}}(1-R) H_{y \mathrm{n}}^{(1)}-\int_{0}^{\infty} k_{z} r_{\mathrm{nt}}\left(k_{x}\right) h_{y \mathrm{nt}}^{(1)}\left(k_{x}\right) \mathrm{d} k_{x} \\
& -\int_{0}^{\infty} k_{z} r_{\mathrm{nb}}\left(k_{x}\right) h_{y \mathrm{nb}}^{(1)}\left(k_{x}\right) \mathrm{d} k_{x} \\
& =\int_{0}^{\infty} k_{z} t_{\mathrm{gt}}\left(k_{x}\right) h_{y \mathrm{gt}}^{(1)}\left(k_{x}\right) \mathrm{d} k_{x}
\end{aligned}
$$

and

$$
\begin{aligned}
& k_{z \mathrm{np}}(1-R) H_{y \mathrm{n}}^{(2,3)}-\int_{0}^{\infty} k_{z} r_{\mathrm{nt}}\left(k_{x}\right) h_{y \mathrm{nt}}^{(2,3)}\left(k_{x}\right) \mathrm{d} k_{x} \\
& -\int_{0}^{\infty} k_{z} r_{\mathrm{nb}}\left(k_{x}\right) h_{y \mathrm{nb}}^{(2,3)}\left(k_{x}\right) \mathrm{d} k_{x} \\
& =k_{z \mathrm{gp}} T H_{y \mathrm{~g}}^{(2,3)}+\int_{0}^{\infty} k_{z} t_{\mathrm{gb}}\left(k_{x}\right) h_{y \mathrm{gb}}^{(2,3)}\left(k_{x}\right) \mathrm{d} k_{x}
\end{aligned}
$$

where $k_{z \text { np }}$ and $k_{z g p}$ are the plasmon wavenumbers in the non-gated and gated waveguides, respectively.

\section{REFLECTION AND TRANSMISSION OF PLASMONS}

Equations (18)-(21) are analogous to the boundary conditions for open waveguides [19], [20], and can be solved by the same methods. This section considers two such methods: a variational and a mode-matching one. It then compares the results to each other and to finite-element simulations.

\section{A. Variational solution and analytical approximations}

Variational methods are based on making a plausible guess for the fields at a junction, and they are well established both for open [20] and closed [21] waveguides. Our interest here is in approximate analytical solutions, for which we need a simple expression for the magnetic field, $\mathcal{H}_{y}$, at the junction between the plasmonic waveguides. Because the power radiated at the junction should be small, we can take $\mathcal{H}_{y}$ as a combination of two plasmons

$$
\mathcal{H}_{y}=C_{\mathrm{n}} H_{y \mathrm{n}}+C_{\mathrm{g}} H_{y \mathrm{~g}}
$$

where $C_{\mathrm{n}}$ and $C_{\mathrm{g}}$ are unknowns, found by substituting Eq. (22) into Eqs. (18)-(21). So, substituting into Eqs. (18) and (19), multiplying by $H_{y \mathrm{n}}$, integrating over $x$, and using the orthogonality conditions gives for the reflection coefficient

$$
\begin{aligned}
& (1+R) S_{\mathrm{np}}=\left\langle H_{y \mathrm{n}} \mathcal{H}_{y}\right\rangle=C_{\mathrm{n}}\left\langle H_{y \mathrm{n}} H_{y \mathrm{n}}\right\rangle \\
& +C_{\mathrm{g}}\left\langle H_{y \mathrm{n}} H_{y \mathrm{~g}}\right\rangle=C_{\mathrm{n}} S_{\mathrm{np}}+C_{\mathrm{g}} J_{\mathrm{pp}}
\end{aligned}
$$

On the other hand, multiplying by $H_{y g}$, integrating and using the orthogonality conditions gives for the transmission coefficient

$$
T S_{\mathrm{gp}}=C_{\mathrm{n}} J_{\mathrm{pp}}+C_{\mathrm{g}} S_{\mathrm{gp}}
$$

Expressing similarly the bulk-mode transmission and reflection coefficients from Eqs. (18) and (19) and substituting the results

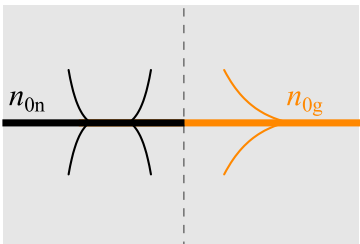

Fig. 5. If the plasmons are confined strongly (which can occur when the frequency is sufficiently high), the gate and air can be ignored. The configuration of Fig. 1(a) can be seen as two non-gated channels embedded in a homogeneous dielectric.

into Eqs. (20) and (21) gives two linear algebraic equations of the form

$$
\begin{aligned}
& C_{\mathrm{n}}\left(1+\frac{k_{z \mathrm{gp}} J_{\mathrm{pp}}^{2}}{k_{z \mathrm{np}} S_{\mathrm{np}} S_{\mathrm{gp}}}+\int_{0}^{\infty} \frac{k_{z} J_{\mathrm{pt}}^{2} \mathrm{~d} k_{x}}{k_{z \mathrm{np}} S_{\mathrm{np}} S_{\mathrm{gtt}}}\right. \\
& \left.+\int_{0}^{\infty} \frac{k_{z} J_{\mathrm{pb}}^{2} \mathrm{~d} k_{x}}{k_{z \mathrm{np}} S_{\mathrm{np}} S_{\mathrm{gbb}}}\right)+C_{\mathrm{g}} \frac{\left(k_{z \mathrm{np}}+k_{z \mathrm{gp}}\right) J_{\mathrm{pp}}}{k_{z \mathrm{np}} S_{\mathrm{np}}}=2 \\
& C_{\mathrm{n}} \frac{k_{z \mathrm{np}}+k_{z \mathrm{gp}}}{k_{z \mathrm{np}}}+C_{\mathrm{g}}\left(\frac{J_{\mathrm{pp}}}{S_{\mathrm{np}}}+\frac{k_{z \mathrm{gp}} S_{\mathrm{gp}}}{k_{z \mathrm{np}} J_{\mathrm{pp}}}\right. \\
& \left.+\int_{0}^{\infty} \frac{k_{z} f_{\mathrm{t}} J_{\mathrm{tp}} \mathrm{d} k_{x}}{k_{z \mathrm{np}} J_{\mathrm{pp}}}+\int_{0}^{\infty} \frac{k_{z} f_{\mathrm{b}} J_{\mathrm{bp}} \mathrm{d} k_{x}}{k_{z \mathrm{np}} J_{\mathrm{pp}}}\right)=2
\end{aligned}
$$

where

$$
\begin{gathered}
f_{\mathrm{t}}=\frac{J_{\mathrm{tp}} S_{\mathrm{nbb}}-J_{\mathrm{bp}} S_{\mathrm{ntb}}}{S_{\mathrm{ntt}} S_{\mathrm{nbb}}-S_{\mathrm{ntb}}^{2}} \\
f_{\mathrm{b}}=\frac{J_{\mathrm{bp}} S_{\mathrm{ntt}}-J_{\mathrm{tp}} S_{\mathrm{ntb}}}{S_{\mathrm{ntt}} S_{\mathrm{nbb}}-S_{\mathrm{ntb}}^{2}}
\end{gathered}
$$

Finding $C_{\mathrm{n}}$ and $C_{\mathrm{g}}$ from Eq. (25) yields, using Eqs. (23) and (24), the reflection and transmission coefficients.

1) Two non-gated channels: The above results can be simplified even further. If the plasmons are confined tightly to the channel (e.g., at high frequencies), neither the gate for the gated nor the air for the non-gated waveguide should play a role. The configuration of Fig. 1(a) can be then replaced by two non-gated channels in a homogeneous dielectric, see Fig. 5. The plasmon fields are then proportional to $\exp \left(-k_{z \mathrm{np}}|x|\right)$ and $\exp \left(-k_{z \mathrm{gp}}|x|\right)$. For them, $S_{\mathrm{np}}=1 /\left(2 k_{z \mathrm{np}}\right)$ and $J_{\mathrm{pp}}=$ $1 /\left(k_{z \mathrm{np}}+k_{z \mathrm{gp}}\right)$. Also, if $k_{z \mathrm{np}} \approx k_{z \mathrm{gp}}$ ('small steps'), a common approximation is to take the variational field at the junction simply as that of the incident wave, $\mathcal{H}_{y}=C_{\mathrm{n}} H_{y \text { n }}$ [33], [34]. Then

$$
\begin{aligned}
& R=\frac{\left(k_{z \mathrm{np}}+k_{z \mathrm{gp}}\right)^{2}-4 k_{z \mathrm{gp}}^{2}}{\left(k_{z \mathrm{np}}+k_{z \mathrm{gp}}\right)^{2}+4 k_{z \mathrm{gp}}^{2}} \\
& T=\frac{4\left(k_{z \mathrm{np}}+k_{z \mathrm{gp}}\right) k_{z \mathrm{gp}}}{\left(k_{z \mathrm{np}}+k_{z \mathrm{gp}}\right)^{2}+4 k_{z \mathrm{gp}}^{2}}
\end{aligned}
$$

These expressions conserve power: the power of the incident plasmon is equal to the sum of the powers of the reflected and transmitted plasmons $\left(|R|^{2}+|T|^{2}=1\right)$. In terms of the transmission-line model [13], [14], [29], the condition $k_{z \mathrm{np}} \approx k_{z \mathrm{gp}}$ means small impedance mismatch between the two waveguides. 

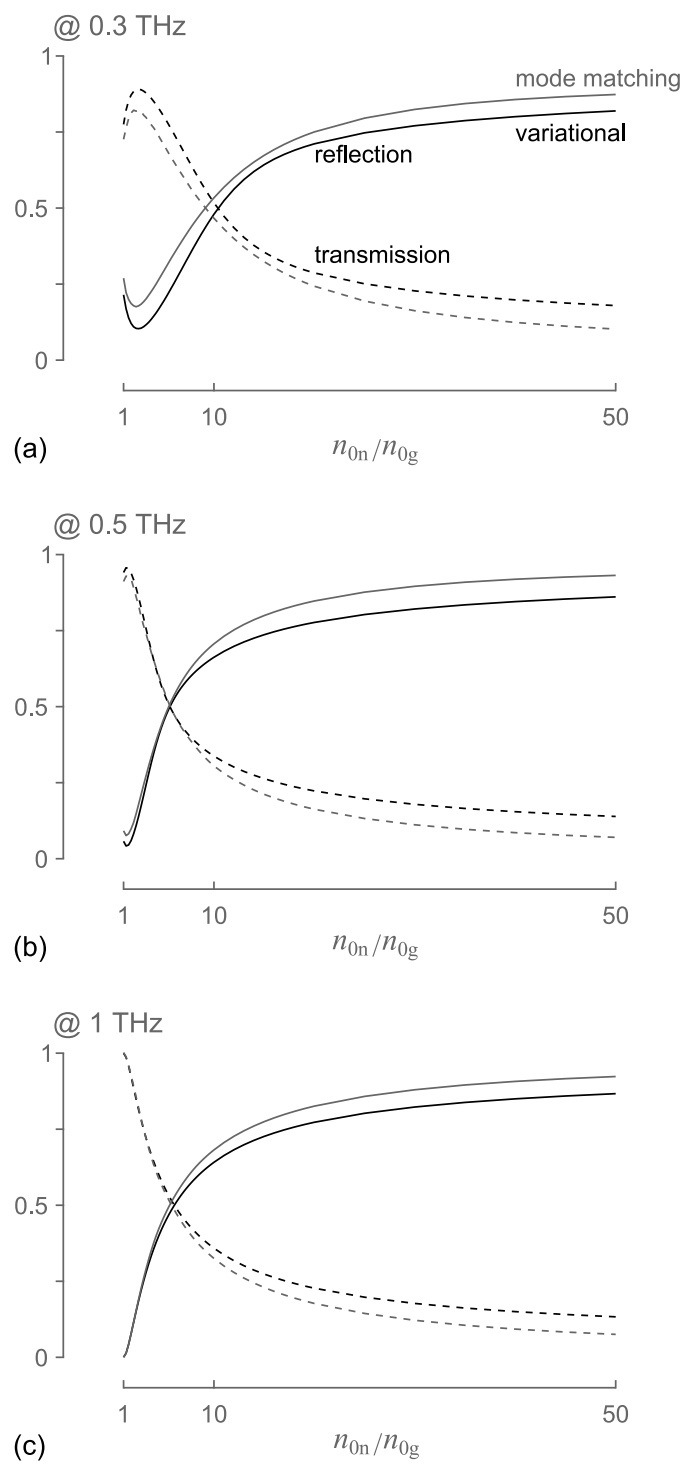

Fig. 6. The power reflection, $\mathcal{R}$, and transmission, $\mathcal{T}$, coefficients depend on the ratio of the dc electron densities, $n_{0 \mathrm{n}} / n_{0 \mathrm{~g}}$. For larger ratios, most of the power reflects back to the non-gated waveguide at all three frequencies. The variational (black lines) and mode-matching (gray lines) solutions agree better at higher frequencies, (b) and (c).

2) Effects of dc electron density: Because the gated dispersion depends on the dc electron density, see Fig. 3, the plasmon reflection and transmission can be controlled by a dc gate voltage. The black lines in Fig. 6 show the plasmon power reflection (solid lines) and transmission (dashed lines) coefficients, $\mathcal{R}$ and $\mathcal{T}$, depending on the ratio $n_{0 \mathrm{n}} / n_{0 \mathrm{~g}}$ at three frequencies. The plasmon powers are calculated using the expressions for the fields, Eqs. (6) and (8), so that, for example, the plasmon reflected power is $|R|^{2} / 2 \operatorname{Re} \int_{-\infty}^{\infty} H_{y \mathrm{n}} E_{x \mathrm{n}}^{*} \mathrm{~d} x$.

Figure 6(c), for $1 \mathrm{THz}$, is the easiest to understand. At such a high frequency, the plasmons are confined to the channel, so that the configuration can be approximated by that of Fig. 5. If the electron densities in both channels are the same, $n_{0 \mathrm{n}} / n_{0 \mathrm{~g}}=1$, there is no junction; the reflection coefficient is

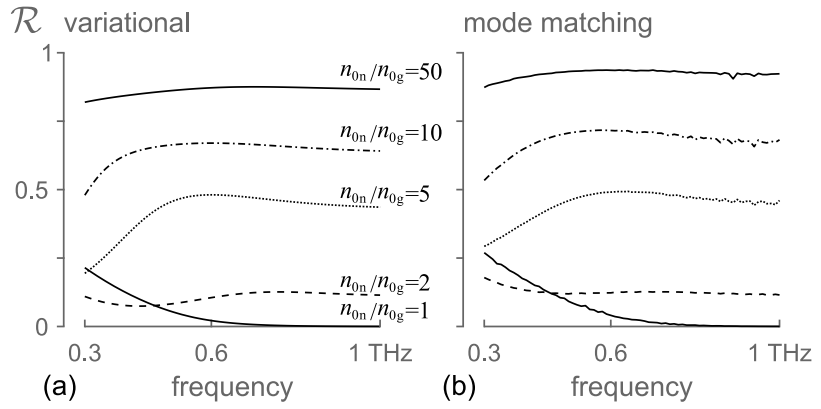

Fig. 7. The power reflection coefficient depends on frequency and is different for different ratios of the dc electron densities, $n_{0 \mathrm{n}} / n_{0 \mathrm{~g}}$. The variational (a) and mode-matching solutions (b) agree.

zero, the transmission is unity. However, as $n_{0 \mathrm{~g}}$ decreases, the difference between the waveguides and, hence, the reflection coefficient increase. At the limit $n_{0 \mathrm{~g}}=0$, the gated channel is empty and supports no plasmons. The incident plasmon should reflect back with $\mathcal{R}=1$. Across a wide frequency range below $1 \mathrm{THz}$, the plasmons behave similarly. For example, the reflection and transmission coefficients at $0.5 \mathrm{THz}$, Fig. 6(b), differ from those at $1 \mathrm{THz}$ only when $n_{0 \mathrm{~g}} \approx n_{0 \mathrm{n}}$.

However, at the lowest frequency of $0.3 \mathrm{THz}$, the qualitative picture of Fig. 5 is invalid, and the plasmon behavior is different, see Fig. 6(b). When $n_{0 \mathrm{n}}=n_{0 \mathrm{~g}}$, about $20 \%$ of plasmon power reflects from the junction. At this frequency, the gate influences strongly the field distribution of the gated plasmons. As a result, the overlap between the gated and nongated plasmons is smaller and the reflection is higher than at the higher frequencies. As $n_{0 \mathrm{~g}}$ decreases, the plasmon reflection coefficient first decreases, reaches a minimum around $n_{0 \mathrm{n}} / n_{0 \mathrm{~g}}=2$, and only then increases. The minimum can be seen as a result of a compensation of the plasmonic mismatch due to the gate by a mismatch of the electron densities. At low values of $n_{0 \mathrm{~g}}$, it tends to unity, like in Figs. 6(b) and (c).

The numerical calculations for all three Fig. 6 conserve power $(\mathcal{R}+\mathcal{T}=1)$ with maximum $1 \%$ relative error and with better accuracy at higher frequencies. As they also show, the power radiated at the junction is negligible. This power is calculated integrating the fields of the bulk modes. In these calculations, the upper limits of the integrals in Eq. (25) were replaced by large finite values.

3) Effects of frequency: The dc electron densities in both channel will determine how the reflection and transmission coefficients depend on frequency, as Fig. 7(a) shows for $\mathcal{R}$ and five values of $n_{0 \mathrm{n}} / n_{0 \mathrm{~g}}: 1,2,5,10$ and 50. Above 0.6 $\mathrm{THz}, \mathcal{R}$ changes little with frequency, and is larger for larger values of $n_{0 \mathrm{n}} / n_{0 \mathrm{~g}}$. The behavior is more complicated at lower frequencies, where the geometry plays a more important role. For example, $\mathcal{R}$ decreases with frequency for $n_{0 \mathrm{n}} / n_{0 \mathrm{~g}}=1$ but increases for $n_{0 \mathrm{n}} / n_{0 \mathrm{~g}}=5$.

In contrast to the power reflection coefficient, $\mathcal{R}$, the amplitude one, $R$, has a complex value. Both its real and imaginary parts change with frequency, as the black lines in Fig. 8 show for $n_{0 \mathrm{n}} / n_{0 \mathrm{~g}}=1$ and 5 .

4) Effects of geometry: By solving Maxwell's equations in the whole plane of the junction, the mode-decomposition 


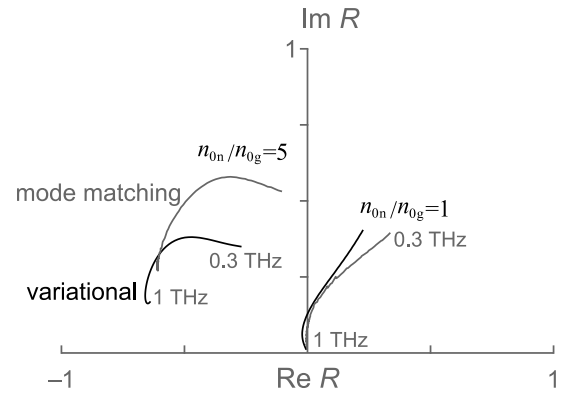

Fig. 8. The amplitude reflection coefficient is complex, and both its real and imaginary parts depend on frequency. The agreement between the variational (black lines) and mode-matching (gray lines) solutions is better for higher frequencies and the lower value of $n_{0 \mathrm{n}} / n_{0 \mathrm{~g}}$.

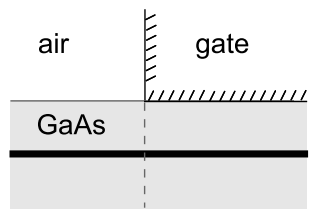

(a)

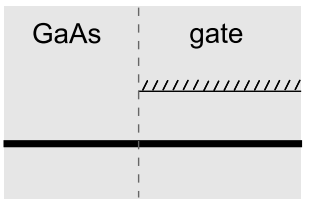

(c)
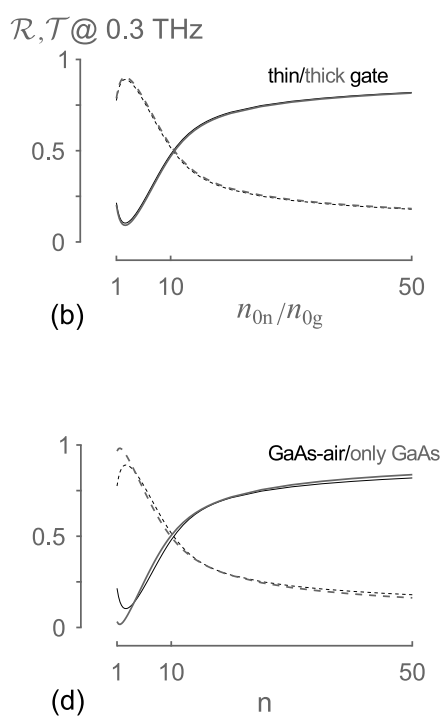

Fig. 9. The mode-decomposition approach takes geometry into account. For example, an infinitely thick gate (a) can be considered instead of the thin gate of Fig. 1(a). However, the power reflection and transmission coefficients (b) are almost the same for the thin (black lines) and thick (gray lines) gates. Also, a homogeneous dielectric (GaAs) can be considered instead of air (c) The dielectric-air interface modifies the power reflection and transmission coefficients at low values of $n_{0 \mathrm{n}} / n_{0 \mathrm{~g}}$ (d).

approach can consider various geometries.

For example, the boundary conditions Eqs. (18)-(21) and the results in Figs. 6-8 were obtained for a gate of zero thickness. These can, however, be easily changed for a gate of infinite thickness, see Fig. 9(a). It is done by replacing the boundary conditions for $x>d$ at the junction with $E_{x}=0$; Eq. (18) is then omitted and the right-hand-side of Eq. (20) is replaced by zero. The results for the reflection and transmission coefficients, however, hardly change, as Fig. 9(b) shows for $0.3 \mathrm{THz}$. The black lines for the infinitely thin gate almost coincide with the gray lines for the infinitely thick one.

We also considered effects of the dielectric-air interface. As Fig. 2 has already shown, the interface modifies the dispersion relation. It also affects the transmission and reflection coefficients, see Fig. 9(d). At low values of $n_{0 \mathrm{n}} / n_{0 \mathrm{~g}}$ the coefficients are different depending on whether there is air or GaAs above $x=d$.

\section{B. Mode-matching solution}

The above variational approach gave analytical expressions for the reflection and transmission coefficients. The cost, however, is the simplified approximation of the junction field, Eq. (22), that can be inaccurate for arbitrary junctions. More accurate methods exist, of which we discuss below the modematching one.

Going back to the boundary conditions Eqs. (18)-(21), the dependence on the coordinate $x$ can be eliminated by multiplying the equations by the fields of plasmons and bulk modes, and integrating over $x$. Mode orthogonality simplifies the expressions, so that the boundary condition for the magnetic field gives the following equations

$$
\begin{aligned}
(1+R) S_{\mathrm{np}} & =T J_{\mathrm{pp}} \\
& +\int_{0}^{\infty} t_{\mathrm{gt}}\left(k_{x}\right) J_{\mathrm{pt}}\left(k_{z \mathrm{np}}, k_{x}\right) \mathrm{d} k_{x} \\
& +\int_{0}^{\infty} t_{\mathrm{gb}}\left(k_{x}\right) J_{\mathrm{pb}}\left(k_{z \mathrm{np}}, k_{x}\right) \mathrm{d} k_{x}
\end{aligned}
$$

and

$$
\begin{aligned}
& r_{\mathrm{nt}}\left(\tilde{k}_{x \mathrm{a}}\right) S_{\mathrm{ntt}}\left(\tilde{k}_{x \mathrm{a}}\right)+r_{\mathrm{nb}}\left(\tilde{k}_{x \mathrm{~d}}\right) S_{\mathrm{ntb}}\left(\tilde{k}_{x \mathrm{~d}}\right) \\
& =T J_{\mathrm{tp}}\left(\tilde{k}_{x \mathrm{a}}, k_{z \mathrm{gp}}\right)+\int_{0}^{\infty} t_{\mathrm{gt}}\left(k_{x}\right) J_{\mathrm{tt}}\left(\tilde{k}_{x \mathrm{a}}, k_{x}\right) \mathrm{d} k_{x} \\
& +\int_{0}^{\infty} t_{\mathrm{gb}}\left(k_{x}\right) J_{\mathrm{tb}}\left(\tilde{k}_{x \mathrm{a}}, k_{x}\right) \mathrm{d} k_{x} \\
& r_{\mathrm{nt}}\left(\tilde{k}_{x \mathrm{a}}\right) S_{\mathrm{ntb}}\left(\tilde{k}_{x \mathrm{a}}\right)+r_{\mathrm{nb}}\left(\tilde{k}_{x \mathrm{~d}}\right) S_{\mathrm{nbb}}\left(\tilde{k}_{x \mathrm{~d}}\right) \\
& =T J_{\mathrm{bp}}\left(\tilde{k}_{x \mathrm{~d}}, k_{z \mathrm{gp}}\right)+\int_{0}^{\infty} t_{\mathrm{gt}}\left(k_{x}\right) J_{\mathrm{bt}}\left(\tilde{k}_{x \mathrm{~d}}, k_{x}\right) \mathrm{d} k_{x} \\
& +\int_{0}^{\infty} t_{\mathrm{gb}}\left(k_{x}\right) J_{\mathrm{bb}}\left(\tilde{k}_{x \mathrm{~d}}, k_{x}\right) \mathrm{d} k_{x}
\end{aligned}
$$

and

$$
\begin{aligned}
& (1+R) J_{\mathrm{pp}}+\int_{0}^{\infty} r_{\mathrm{nt}}\left(k_{x}\right) J_{\mathrm{tp}}\left(k_{x}, k_{z \mathrm{gp}}\right) \mathrm{d} k_{x} \\
& +\int_{0}^{\infty} r_{\mathrm{nb}}\left(k_{x}\right) J_{\mathrm{bp}}\left(k_{x}, k_{z \mathrm{gp}}\right) \mathrm{d} k_{x}=T S_{\mathrm{gp}}
\end{aligned}
$$

and

$$
\begin{aligned}
& (1+R) J_{\mathrm{pt}}\left(k_{z \mathrm{np}}, \tilde{k}_{x \mathrm{a}}\right)+\int_{0}^{\infty} r_{\mathrm{nt}}\left(k_{x}\right) J_{\mathrm{tt}}\left(k_{x}, \tilde{k}_{x \mathrm{a}}\right) \mathrm{d} k_{x} \\
& +\int_{0}^{\infty} r_{\mathrm{nb}}\left(k_{x}\right) J_{\mathrm{bt}}\left(k_{x}, \tilde{k}_{x \mathrm{a}}\right) \mathrm{d} k_{x}=t_{\mathrm{gt}}\left(\tilde{k}_{x \mathrm{a}}\right) S_{\mathrm{gtt}}\left(\tilde{k}_{x \mathrm{a}}\right) \\
& (1+R) J_{\mathrm{pb}}\left(k_{z \mathrm{np}}, \tilde{k}_{x \mathrm{~d}}\right)+\int_{0}^{\infty} r_{\mathrm{nt}}\left(k_{x}\right) J_{\mathrm{tb}}\left(k_{x}, \tilde{k}_{x \mathrm{~d}}\right) \mathrm{d} k_{x} \\
& +\int_{0}^{\infty} r_{\mathrm{nb}}\left(k_{x}\right) J_{\mathrm{bb}}\left(k_{x}, \tilde{k}_{x \mathrm{~d}}\right) \mathrm{d} k_{x}=t_{\mathrm{gb}}\left(\tilde{k}_{x \mathrm{~d}}\right) S_{\mathrm{gbb}}\left(\tilde{k}_{x \mathrm{~d}}\right)
\end{aligned}
$$

On the other hand, the boundary condition for the electric field gives the following equations

$$
\begin{aligned}
(1-R) k_{z \mathrm{np}} S_{\mathrm{np}} & =k_{z \mathrm{gp}} T J_{\mathrm{pp}} \\
& +\int_{0}^{\infty} k_{z} t_{\mathrm{gt}}\left(k_{x}\right) J_{\mathrm{pt}}\left(k_{z \mathrm{np}}, k_{x}\right) \mathrm{d} k_{x} \\
& +\int_{0}^{\infty} k_{z} t_{\mathrm{gb}}\left(k_{x}\right) J_{\mathrm{pb}}\left(k_{z \mathrm{np}}, k_{x}\right) \mathrm{d} k_{x}
\end{aligned}
$$


and

$$
\begin{aligned}
& -\tilde{k}_{z} r_{\mathrm{nt}}\left(\tilde{k}_{x \mathrm{a}}\right) S_{\mathrm{ntt}}\left(\tilde{k}_{x \mathrm{a}}\right)-\tilde{k}_{z} r_{\mathrm{nb}}\left(\tilde{k}_{x \mathrm{~d}}\right) S_{\mathrm{ntb}}\left(\tilde{k}_{x \mathrm{~d}}\right) \\
& =T k_{z \mathrm{gp}} J_{\mathrm{tp}}\left(\tilde{k}_{x \mathrm{a}}, k_{z \mathrm{gp}}\right)+\int_{0}^{\infty} k_{z} t_{\mathrm{gt}}\left(k_{x}\right) J_{\mathrm{tt}}\left(\tilde{k}_{x \mathrm{a}}, k_{x}\right) \mathrm{d} k_{x} \\
& +\int_{0}^{\infty} k_{z} t_{\mathrm{gb}}\left(k_{x}\right) J_{\mathrm{tb}}\left(\tilde{k}_{x \mathrm{a}}, k_{x}\right) \mathrm{d} k_{x} \\
& -\tilde{k}_{z} r_{\mathrm{nt}}\left(\tilde{k}_{x \mathrm{a}}\right) S_{\mathrm{ntb}}\left(\tilde{k}_{x \mathrm{a}}\right)-\tilde{k}_{z} r_{\mathrm{nb}}\left(\tilde{k}_{x \mathrm{~d}}\right) S_{\mathrm{nbb}}\left(\tilde{k}_{x \mathrm{~d}}\right) \\
& =T k_{z \mathrm{gp}} J_{\mathrm{bp}}\left(\tilde{k}_{x \mathrm{~d}}, k_{z \mathrm{gp}}\right)+\int_{0}^{\infty} k_{z} t_{\mathrm{gt}}\left(k_{x}\right) J_{\mathrm{bt}}\left(\tilde{k}_{x \mathrm{~d}}, k_{x}\right) \mathrm{d} k_{x} \\
& +\int_{0}^{\infty} k_{z} t_{\mathrm{gb}}\left(k_{x}\right) J_{\mathrm{bb}}\left(\tilde{k}_{x \mathrm{~d}}, k_{x}\right) \mathrm{d} k_{x}
\end{aligned}
$$

and

$$
\begin{aligned}
& k_{z \mathrm{np}}(1-R) J_{\mathrm{pp}}-\int_{0}^{\infty} k_{z} r_{\mathrm{nt}}\left(k_{x}\right) J_{\mathrm{tp}}\left(k_{x}, k_{z \mathrm{gp}}\right) \mathrm{d} k_{x} \\
& -\int_{0}^{\infty} k_{z} r_{\mathrm{nb}}\left(k_{x}\right) J_{\mathrm{bp}}\left(k_{x}, k_{z \mathrm{gp}}\right) \mathrm{d} k_{x}=k_{z \mathrm{gp}} T S_{\mathrm{gp}}
\end{aligned}
$$

and

$$
\begin{aligned}
& k_{z \mathrm{np}}(1-R) J_{\mathrm{pt}}\left(k_{z \mathrm{np}}, \tilde{k}_{x \mathrm{a}}\right)-\int_{0}^{\infty} k_{z} r_{\mathrm{nt}}\left(k_{x}\right) J_{\mathrm{tt}}\left(k_{x}, \tilde{k}_{x \mathrm{a}}\right) \mathrm{d} k_{x} \\
& -\int_{0}^{\infty} k_{z} r_{\mathrm{nb}}\left(k_{x}\right) J_{\mathrm{bt}}\left(k_{x}, \tilde{k}_{x \mathrm{a}}\right) \mathrm{d} k_{x}=\tilde{k}_{z} t_{\mathrm{gt}}\left(\tilde{k}_{x \mathrm{a}}\right) S_{\mathrm{gtt}}\left(\tilde{k}_{x \mathrm{a}}\right) \\
& k_{z \mathrm{np}}(1-R) J_{\mathrm{pb}}\left(k_{z \mathrm{np}}, \tilde{k}_{x \mathrm{~d}}\right)-\int_{0}^{\infty} k_{z} r_{\mathrm{nt}}\left(k_{x}\right) J_{\mathrm{tb}}\left(k_{x}, \tilde{k}_{x \mathrm{a}}\right) \mathrm{d} k_{x} \\
& -\int_{0}^{\infty} k_{z} r_{\mathrm{nb}}\left(k_{x}\right) J_{\mathrm{bb}}\left(k_{x}, \tilde{k}_{x \mathrm{~d}}\right) \mathrm{d} k_{x}=\tilde{k}_{z} t_{\mathrm{gb}}\left(\tilde{k}_{x \mathrm{~d}}\right) S_{\mathrm{gbb}}\left(\tilde{k}_{x \mathrm{~d}}\right)
\end{aligned}
$$

The expressions for the overlaps between the bulk modes contain terms proportional to $1 /\left(k_{z}-\tilde{k}_{z}\right)$. This singularity can be avoided by multiplying Eqs. (28) and (30) by $\tilde{k}_{z}$ and adding to or subtracting from Eqs. (33) and (34). The results, together with Eqs. (27), (29), (31) and (33), form a system of coupled integral equations. We solved it numerically, first, by replacing the upper limits of the integrals with a large but finite number and then by discretizing the integrals using the trapezoidal rule. The result is a system of linear algebraic equations for the plasmon reflection and transmission coefficients and for the discretized reflection and transmission coefficients of the bulk modes. We then compared the results of the modematching model with the variational solutions for the range of frequencies and electron densities of Figs. 6-8.

The gray lines in Fig. 6 show the plasmon power coefficients depending on the dc electron density at three frequencies. These behave similarly to the variational solutions (black lines). The reflection coefficients are small at smaller values of $n_{0 \mathrm{n}} / n_{0 \mathrm{~g}}$ and tend to unity as larger values. The agreement between the mode-matching and the variational solutions is better at higher frequencies and lower values of $n_{0 \mathrm{n}} / n_{0 \mathrm{~g}}$. The reason is that the expression for the variational field, Eq. (22), took only the plasmons into account. It is more accurate when the plasmonic mismatch is weak, which occurs at higher frequencies and small values of $n_{0 \mathrm{n}} / n_{0 \mathrm{~g}}$. When the mismatch is strong, the variational solution could be expected to underestimate the reflection. A similar behavior can be seen in Fig. 7(b) that shows the frequency dependence of the power reflection coefficient at several values of $n_{0 \mathrm{n}} / n_{0 \mathrm{~g}}$. Like for Fig. 6, the mode-matching solutions are close to the variational ones, see Fig. 7(a), although their values of $\mathcal{R}$ are generally higher. In both Fig. 6 and Fig. 7(b), the modematching solution conserves power $\mathcal{R}+\mathcal{T}=1$.

The values of the amplitude coefficients, $R$ and $T$, are complex, as the dashed lines in Fig. 8 show for the reflection coefficient. However, the phases given by the mode-matching and the variational (solid lines) solutions agree less well than their amplitudes, especially for the higher value of $n_{0 \mathrm{n}} / n_{0 \mathrm{~g}}$. A possible reason is the approximate expression of variational field, Eq. (22), which tends to underestimate the effects of the non-plasmonic modes. These modes carry negligible power and should have a limited effect on the amplitudes of the reflection and transmission coefficient. They have, however, a greater effect on the phases, as Fig. 8 shows.

In addition to the limitations of the variational solution, the entire mode-matching approach of this paper uses several approximations. First, it ignored plasmonic loss, which is valid at low temperatures and sufficiently high electron densities. However, the loss should increase for gated channels with low densities and, therefore, may dominate, in real structures, the reflection and transmission coefficients for large values of $n_{0 \mathrm{n}} / n_{0 \mathrm{~g}}$. Second, our model assumed, as is usually done in the literature, abrupt junctions between gated and non-gated channels. In real devices, the fringing fields of the gate and electron diffusion will create a continuous transition of the electron concentration from $n_{0 \mathrm{n}}$ to $n_{0 \mathrm{~g}}$. Continuous transitions are often used as a means to match waveguides and reduce reflections, see e.g. [35]. And while the magnitude of the effect in real devices would require an additional study, one could expect that the assumption of an abrupt junction might overestimate the plasmonic reflection coefficient.

As stated above, when a plasmon is incident on a junction, the power radiated from the junction (i.e. the power converted into continuous modes with real wavenumbers) is negligible. This result could be expected: the plasmon wavenumbers in our configurations exceed the radiative-mode wavenumbers by two orders of magnitude, and the overlap between the plasmons and the radiative modes is small. The fields are matched at the interfaces by exciting the evanescent modes, which have higher wavenumbers but do not carry power. This result also agrees with experimental observations. Ever since the early work of Allen et al. [36], experimentalists have been using special structures, such as grating couplers [37]-[40] and antennas [13], [14], [41], to couple electromagnetic radiation to plasmons. In particular, grating couplers create a series of alternating gated and non-gated channels, and can couple plasmons to electromagnetic radiation due to their periodicity. It is in contrast to the single non-periodic junctions considered here.

Although we concentrate here on junctions between gated and non-gated channels, the mode-matching techniques are also applicable to other geometries [27]. Practically important are, in particular, contacts to the channels. Ideal, perfectly conducting, contacts can be characterized by the boundary condition $E_{x}=0$. On the other hand, more realistic contacts can be characterized by impedance boundary conditions of the 

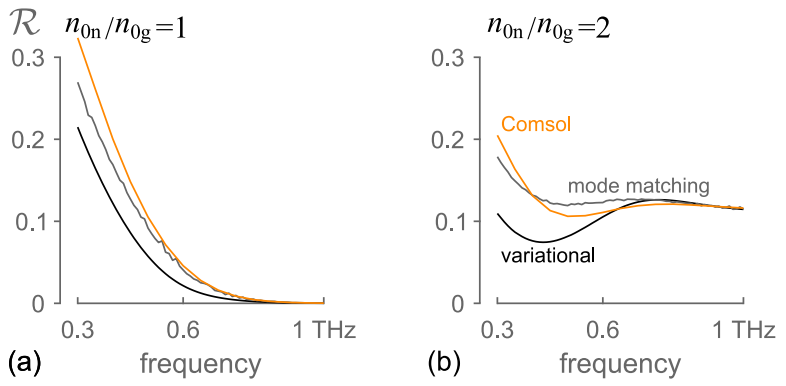

Fig. 10. The solutions by the finite-element method (orange lines) behave similarly to those by the variational (black lines) and mode-matching (gray lines) methods for $n_{0 \mathrm{n}} / n_{0 \mathrm{~g}}=1$ (a) and $n_{0 \mathrm{n}} / n_{0 \mathrm{~g}}=2$ (b). The agreement is better between the finite-element and the mode-matching methods.

form $E_{x}=Z_{\mathrm{s}} H_{y}$. Contacts constitute device boundaries and so should be analyzed when considering a complete device, which will be a subject of future work.

\section{Comparison with the finite-element method}

We also calculated the reflection coefficient using the frequency-domain finite-element package Comsol Multiphysics. The channels were described as a thin $(20 \mathrm{~nm})$ threedimensional plasma slab with a Drude permittivity. The electron density of the slab corresponded to the two-dimensional density of the channels used above. The gate was modelled as a $10 \mathrm{~nm}$ thick perfect electric conductor. To close the computational domain, two perfect electric conductors were placed at $20 \mu \mathrm{m}$ above and below the channels. This distance was large enough not to affect plasmons. The other parameters were the same as for the mode-matching analysis.

The calculations had two steps. First, the eigenmode solver determined the dispersion of the gated and non-gated plasmons. They agreed excellently with the theoretical ones, see Fig. 2 and 3. Then, a plasmon was launched from the nongated waveguide, the total field computed, and the reflection coefficient evaluated.

Figure 10 compares the results with the above solutions for the power reflection coefficient. All three solutions are qualitatively similar both for $n_{0 \mathrm{n}} / n_{0 \mathrm{~g}}=1$ and 2 . The Comsol results (orange lines) are closer to the mode-matching solutions (gray lines) than to the variational solutions (black lines), which might be due to the approximations of the latter.

\section{Effects of electron collisions}

The lossless equation of motion Eq. (1) describes well the plasmons observed experimentally at liquid-helium temperatures, see e.g. [42], [43]. At higher temperatures, electron collisions will play a role depending on the relationship between the angular frequency $\omega$ and the collision frequency $\tau$. For $\omega \tau \lesssim 1$, the mode profiles, and thus the reflection and transmission coefficients, will differ considerably from the the lossless ones discussed here. On the other hand, for $\omega \tau \gg 1$, the mode profiles will be little affected by loss, similar to the situation in lossy dielectric waveguides [44]. As a result, the reflection and transmission coefficients will remain almost the same, and the effect of collisions will be limited to propagation loss.

To check this qualitative picture, we employed the finiteelement model of Sec. IV-C. The loss was taken into account by a collision frequency $\gamma=1 / \tau$ in the Drude permittivity. We then repeated the calculations for $\gamma=10^{11} \mathrm{rad} / \mathrm{s}$; the corresponding values of $\omega \tau$ changed from around 20 to 60 between 0.3 and $1 \mathrm{THz}$, and the electron mobility was of the order of $10^{5} \mathrm{~cm}^{2} /(\mathrm{V} \cdot \mathrm{s})$. After accounting for the propagation loss, we could calculate the values of $\mathcal{R}$. In agreement with our expectations, they did not change appreciably compared to those of Fig. 10. Analogous results were previously obtained for the related problem of lossy plasmons reflecting from contacts in the presence of dc currents [26].

\section{CONCLUSIONS}

A plasmon will partially reflect and transmit when incident upon a junction between a gated and non-gated channels. Also, it will excite bulk radiation and evanescent modes. As this paper has shown, the plasmon reflection and transmission can be studied by variational and mode-matching methods. The aim of the variational method was to obtain analytical expressions, whereas the mode-matching one gave numerical solutions.

The behavior of the plasmon reflection and transmission coefficients depended on the frequency. At higher frequencies, the plasmons were confined tightly to the channels. As a result, the effects of geometry, such as the air-dielectric interface and the gate, could be ignored. The channels can be seen as non-gated ones surrounded by a homogeneous dielectric. In this regime, the plasmon reflection coefficient could be increased by increasing the difference between the channel electron densities. At lower frequencies, however, the situation was more complicated. For example, the reflection coefficient could increase or decrease with frequency depending on the electron density. The analytical expressions appeared to be more accurate at higher frequencies, and both the variational and the mode-matching methods agreed with finite-element calculations.

As the results show, mode decomposition is a powerful method to study plasmons at junctions between gated and non-gated channels. On one hand, the method is rigorous; it relies on solving Maxwell's equations and can tackle various geometries. On the other hand, it is easy to implement, fast, and can even result in simple analytical approximations. These results can be used further for the design of devices combining several junctions, for example, plasmonic crystals, resonators, detectors, and oscillators.

\section{ACKNOWLEDGMENT}

O.S. is grateful to R. R. A. Syms and V. V. Popov for valuable discussions. The work at Sandia National Laboratories was supported by the DOE Office of Basic Energy Sciences. Sandia National Laboratories is a multi-program laboratory managed and operated by Sandia Corporation, a wholly owned subsidiary of Lockheed Martin Corporation, for the U.S. Department of Energy's National Nuclear Security Administration under contract DE-AC04-94AL85000. 


\section{REFERENCES}

[1] M. Dyakonov and M. Shur, "Detection, mixing, and frequency multiplication of terahertz radiation by two-dimensional electronic fluid," IEEE Trans. Electron. Dev., vol. 43, no. 3, pp. 380-387, 1996.

[2] G. Dyer, G. Aizin, J. Reno, E. Shaner, and S. Allen, "Novel tunable millimeter-wave grating-gated plasmonic detectors," IEEE J. Sel. Top. Quant. Electron., vol. 17, pp. 85-91, 2011.

[3] V. V. Popov, D. V. Fateev, T. Otsuji, Y. M. Meziani, D. Coquillat, and W. Knap, "Plasmonic terahertz detection by a double-grating-gate fieldeffect transistor structure with an asymmetric unit cell," Appl. Phys. Lett., vol. 99, p. 243504, 2011.

[4] V. M. Muravev and I. V. Kukushkin, "Plasmonic detector/spectrometer of subterahertz radiation based on two-dimensional electron system with embedded defect," Appl. Phys. Lett., vol. 100, p. 082102, 2012.

[5] M. Dyakonov and M. Shur, "Shallow water analogy for a ballistic fieldeffect transistor: new mechanism of plasma wave generation by a dc current," Phys. Rev. Lett., vol. 71, pp. 2465-2468, 1993.

[6] F. J. Crowne, "Contact boundary conditions and the Dyakonov-Shur instability in high electron mobility transistors," J. Appl. Phys., vol. 82, pp. 1242-1254, 1997.

[7] M. Dyakonov and M. S. Shur, "Current instability and plasma waves generation in ungated two-dimensional electron layers," Appl. Phys. Lett., vol. 87, no. 11, p. 111501, 2005.

[8] O. Sydoruk, V. Kalinin, and L. Solymar, "Terahertz instability of optical phonons interacting with plasmons in two-dimensional electron channels," Appl. Phys. Lett., vol. 97, pp. 062 107-1-3, 2010.

[9] O. Sydoruk, R. R. A. Syms, and L. Solymar, "Distributed gain in plasmonic reflectors and its use for terahertz generation," Opt. Express, vol. 20, pp. 19618-19627, 2012 .

[10] M. Lee, M. C. Wanke, and J. L. Reno, "Millimeter wave mixing using plasmon and bolometric response in a double-quantum-well field-effect transistor," Appl. Phys. Lett., vol. 86, no. 3, p. 033501, 2005.

[11] V. Muravev, I. Kukushkin, J. Smet, and K. von Klitzing, "Millimeter/submillimeter mixing based on the nonlinear plasmon response of two-dimensional electron systems," JETP Lett., vol. 90, pp. 197-201, 2009.

[12] N. M. T. Nishimura and T. Otsuji, "An intensity modulator for terahertz electromagnetic waves utilizing two-dimensional plasmon resonance in a dual-grating-gate high-electron-mobility transistor," Jpn. J. Appl. Phys., vol. 49, p. 054301, 2010.

[13] G. C. Dyer, G. R. Aizin, S. Preu, N. Q. Vinh, S. J. Allen, J. L. Reno, and E. A. Shaner, "Inducing an incipient terahertz finite plasmonic crystal in coupled two dimensional plasmonic cavities," Phys. Rev. Lett., vol. 109 , pp. $126803-1-5,2012$.

[14] G. C. Dyer, G. R. Aizin, S. J. Allen, A. D. Grine, D. Bethke, J. L. Reno, and E. A. Shaner, "Induced transparency by coupling of tamm and defect states in tunable terahertz plasmonic crystals," Nat. Photon., vol. 7, no. 11, pp. 925-930, 112013.

[15] F. Rana, "Graphene terahertz plasmon oscillators," IEEE Trans. Nanotechnol., vol. 7, pp. 91-99, 2008.

[16] T. Otsuji, S. A. Boubanga Tombet, A. Satou, H. Fukidome, M. Suemitsu, E. Sano, V. Popov, M. Ryzhii, and V. Ryzhii, "Graphene-based devices in terahertz science and technology," J. Phys. D: Appl. Phys., vol. 45, pp. $303001-1-9,2012$.

[17] V. Y. Kachorovskii and M. S. Shur, "Current-induced terahertz oscillations in plasmonic crystal," Appl. Phys. Lett., vol. 100, no. 23, pp. 232 108-1-3, 2012.

[18] V. V. Shevchenko, Continuous Transitions in Open Waveguides. Boulder, Colorado: Golem Press, 1971.

[19] D. Marcuse, Light Transmission Optics. New York: Van Nostrand Reinhold, 1982.

[20] T. Rozzi and M. Mongiardo, Open Electromagnetic Waveguides. London: IET, 1996

[21] R. E. Collin, Field Theory of Guided Waves. New York: WileyInterscience-IEEE, 1991.

[22] S. Thongrattanasiri, J. Elser, and V. A. Podolskiy, "Quasi-planar optics: computing light propagation and scattering in planar waveguide arrays," J. Opt. Soc. Am. B, pp. B102-B110, 2009.

[23] S. E. Kocabas, G. Veronis, D. A. B. Miller, and S. Fan, "Modal analysis and coupling in metal-insulator-metal waveguides," Phys. Rev. B, vol. 79, p. 035120, 2009.

[24] O. Sydoruk, R. R. A. Syms, and L. Solymar, "Amplifying mirrors for terahertz plasmons," J. Appl. Phys., vol. 112, p. 104512, 2012.

[25] O. Sydoruk, "Drifting plasmons in open two-dimensional channels: modal analysis," J. Phys. D: Appl. Phys., vol. 46, pp. 135 103-1-8, 2013.
[26] _ "Amplification of drifting semiconductor plasmons and effects of carrier collisions and diffusion," J. Phys. D: Appl. Phys., vol. 46, pp. $345101-1-7,2013$

[27] _ "Amplification and generation of terahertz plasmons in gated twodimensional channels: Modal analysis," J. Appl. Phys., vol. 115, pp. 204 507-1-7, 2014.

[28] V. V. Popov, G. M. Tsymbalov, and M. S. Shur, "Plasma wave instability and amplification of terahertz radiation in field-effect-transistor arrays," J. Phys.: Condens. Matter, vol. 20, pp. 384 208-1-6, 2008.

[29] G. R. Aizin and G. C. Dyer, "Transmission line theory of collective plasma excitations in periodic two-dimensional electron systems: Finite plasmonic crystals and tamm states," Physical Review B, vol. 86, no. 23, p. 235316, 2012.

[30] M. Karabiyik, C. Al-Amin, and N. Pala, "Deep sub-wavelength multimode tunable in-plane plasmonic lenses operating at terahertz frequencies," IEEE Trans. Terahertz Sci. Technol., vol. 3, pp. 550-557, 2013.

[31] M. Khorrami, S. El-Ghazaly, H. Naseem, and S.-Q. Yu, "Global modeling of active terahertz plasmonic devices," EEE Trans. Terahertz Sci. Technol., vol. 4, no. 1, pp. 101-109, 2014.

[32] C. D. Ager, R. J. Wilkinson, and H. P. Hughes, "Periodic gratinggate screening of plasmons in heterojunction structures," J. Appl. Phys., vol. 71, no. 3, pp. 1322-1326, 1992.

[33] D. Marcuse, "Radiation losses of tapered dielectric slab waveguides," Bell Syst. Tech. J., vol. 49, pp. 273-287, 1970.

[34] T. E. Rozzi, "Rigorous analysis of the step discontinuity in a planar dielectric waveguide," IEEE Trans. Microw. Theory Tech., vol. MTT-26, pp. 738-746, 1978.

[35] R. E. Collin, Foundations for Microwave Engineering. Hoboken, New Jersey: Wiley-IEEE Press, 2001.

[36] S. J. Allen, D. C. Tsui, and R. A. Logan, "Observation of the twodimensional plasmon in silicon inversion layers," Phys. Rev. Lett., vol. 38, pp. 980-983, 1977.

[37] T. N. Theis, "Plasmons in inversion layers," Surf. Sci., vol. 98, pp. 515532, 1980.

[38] E. Batke, D. Heitmann, and C. W. Tu, "P1asmon and magnetoplasmon excitation in two-dimensional electron space-charge layers on gaas," Phys. Rev. B, vol. 34, pp. 6951-6960, 1986.

[39] R. J. Wilkinson, C. D. Ager, T. Duffield, H. P. Hughes, D. G. Hasko, H. Ahmed, J. Frost, D. C. Peacock, D. A. Ritchie, G. A. C. Jones, C. R. Whitehouse, and N. Apsley, "Plasmon excitation and selfcoupling in a biperiodically modulated two dimensional electron gas," J. Appl. Phys., vol. 71, pp. 6049-6061, 1992.

[40] N. Sekine, K. Yamanaka, K. Hirakawa, M. Voßebürger, P. HaringBolivar, and H. Kurz, "Observation of terahertz radiation from higherorder two-dimensional plasmon modes in gaas/algaas single quantum wells," Appl. Phys. Lett., vol. 74, pp. 1006-1008, 1999.

[41] A. Di Gaspare, V. Giliberti, E. Giovine, F. Evangelisti, and M. Ortolani, "Spectroscopic study of plasma wave resonances of a two-dimensional electron gas in a microcavity at low temperatures," J. Opt., vol. 15, pp. $114012-1-6,2013$.

[42] D. Olego, A. Pinczuk, A. C. Gossard, and W. Wiegmann, "Plasma dispersion in a layered electron gas: A determination in gaas-(aiga)as heterostructures," Phys. Rev. B, vol. 26, pp. 7867-7870, 1982.

[43] L. C. O. Súilleabháin, H. P. Hughes, A. C. Churchill, D. A. Ritchie, M. P. Grimshaw, and G. A. C. Jones, "Raman studies of plasmon modes in a drifting two-dimensional electron gas," J. Appl. Phys., vol. 76, pp. $1701-1705,1994$

[44] A. W. Snyder and J. D. Love, Optical Waveguide Theory. Dodrecht, Netherlands: Kluwer Academic Publishers, 2000.

Oleksiy Sydoruk Oleksiy Sydoruk is a lecturer with the Department of Electrical and Electronic Engineering, Imperial College London, UK. Prior to that he was a Junior Research Fellow and a Newton Fellow with Imperial College. Prior to that he was a research assistant with Erlangen-Nuremberg and Osnabrueck Universities, Germany. He has published in the fields of metamaterials, polarization optics, and biomedical optics. His current interests are in plasmonics, particularly for applications in terahertz sources. 
Kaushal Choonee Kaushal Choonee received the Ph.D. degree in BioMEMS from the Electrical Engineering Department at Imperial College London, UK. He was subsequently a research associate at the same institution where he investigated the applications of plasmonic fields in micro-scale chemistry. He is currently a senior research scientist at the National Physical Laboratory, Teddington, UK, where he is developing micro-engineered devices for quantum technologies. His interests include MEMS, nanotechnology, and electromagnetic modeling.

Gregory C. Dyer Gregory C. Dyer is presently a Senior Member of Technical Staff at Sandia National Laboratories, USA. Previously he has held positions as a postdoctoral researcher at Sandia National Laboratories and as a graduate student researcher at the University of California, Santa Barbara, USA. He has developed and characterized far infrared detectors, sources and devices, with a focus upon the engineering of quantum-confined semiconductor plasmas for terahertz applications. His current research interests include low-dimensional electronic systems, far infrared devices, plasmonics, photonic crystals, and electromagnetic modeling. 